\title{
Politique
}

\section{Nouveaux groupes sociaux et nouveaux enjeux politiques : exemple des personnes âgées aux États-Unis}

\section{Pierre Noreau}

Numéro 17, hiver 1990

Les nouveaux enjeux du politique

URI : https://id.erudit.org/iderudit/040646ar

DOI : https://doi.org/10.7202/040646ar

Aller au sommaire du numéro

Éditeur(s)

Société québécoise de science politique

ISSN

0711-608X (imprimé)

1918-6584 (numérique)

Découvrir la revue

Citer cet article

Noreau, P. (1990). Nouveaux groupes sociaux et nouveaux enjeux politiques : exemple des personnes âgées aux États-Unis. Politique, (17), 5-29.

https://doi.org/10.7202/040646ar d'utilisation que vous pouvez consulter en ligne.

https://apropos.erudit.org/fr/usagers/politique-dutilisation/ 


\title{
Nouveaux groupes sociaux et nouveaux enjeux politiques: exemple des personnes âgées aux États-Unis
}

\author{
Pierre Noreau \\ Institut d'études politiques de Paris
}

Au cours des 30 dernières années, les sociétés occidentales ont vu se multiplier les nouveaux groupes sociaux et, avec eux, les formes et les moyens d'action collective (Tarrow, 1988). En faisant éclater les appartenances socio-économiques traditionnelles, leur prolifération a non seulement permis la reconstruction du champ social, mais elle a aussi provoqué la multiplication des acteurs politiques organisés (Pizzorno, 1981, p. 268). Aux côtés des «partenaires» sociaux habituels (patronat, organisations syndicales et agricoles, mouvement coopératif), on a vu naître de nouveaux pôles de rassemblement, fondés sur l'identité sexuelle, sur l'origine ethnique ou régionale et sur l'appartenance à une génération ou à une autre, jeunes ou aînés. Le phénomène est d'autant plus intéressant qu'il s'observe dans plusieurs pays occidentaux (Melucci, 1983).

Les sociologues se sont penchés sur ces initiatives organisées, les appelant tantôt «nouveaux mouvements sociaux», tantôt «mouvements post-politiques» (Melucci, 1978). On les a parfois décrites comme l'action collective des nouvelles «minorités» (Bourassa, 1987, p. 98). Les psychosociologues ont pour leur part trai- 
té la question sous l'angle des identités collectives (Weigert et al., 1986). Si la science politique s'y est intéressée, c'est surtout parce que l'action de ces nouveaux groupes sociaux remet en question la légitimité représentative des agents politiques traditionnels, c'est-àdire des instances électives des partis politiques ainsi que des intervenants socio-économiques établis depuis plus longtemps (Lacroix, 1985, p. 182). Plus concrètement, l'apparition de ces groupes sociaux favorise la multiplication des acteurs dans l'espace politique organisé. Elle commande aussi l'établissement de rapports souvent complexes et fonde les bases d'enjeux politiques nouveaux, que nous tenterons d'exposer ici.

Aux États-Unis, pays qui retiendra notre attention, l'action collective des personnes âgées est au nombre des phénomènes qui viennent d'être évoqués. Cette mobilisation récente fournit un exemple intéressant des enjeux que soulève l'action collective des «nouvelles minorités».

Enjeux et acteurs: de quelques catégories

Pour des raisons difficiles à saisir, le concept d'enjeux, pourtant utilisé si souvent en sociologie et en science politique, n'a jamais été défini clairement, même par les auteurs anglosaxons qui s'y réfèrent. On doit à Léon Dion les définitions les plus opératoires. L'enjeu, écrit Dion (1967, p. 21), est un «thème mettant en cause des valeurs et des intérêts que les participants considèrent importants pour eux-mêmes ou pour le bien public». Il souligne ailleurs:

Qu'un enjeu est un objet social qui, par suite d'un concours de circonstances, émerge des débats publics et devient matière à considération par les centres de décision et qui, de ce fait, met en cause des idéologies et des intérêts qu'individus et groupes jugent importants pour eux-mêmes ou pour le bien général, ce qui les conduit fréquemment à se départager en factions opposées conformément à leurs positions respectives (Dion, 1971, p. 88). 
Certains éléments de cette définition sont particulièrement utiles pour la suite de notre propos: l'enjeu comporte une «dimension politique» dans la mesure où il devient l'objet de la considération des centres de décision; il met «en cause» un ou plusieurs «acteurs» en "conflit» (individus ou groupes départagés en factions). L'enjeu est partagé par tous les acteurs concernés. Le problème que pose une situation de fait n'appartient exclusivement, en effet, à aucun des acteurs intéressés.

La sociologie des mouvements sociaux d'Alain Touraine nous apprend par ailleurs qu'il peut exister plusieurs niveaux de conflits, que ces conflits mettent en cause les dimensions différentes d'un même enjeu et font s'opposer divers types d'acteurs: classes sociales, acteurs politiques ou organisationnels (Touraine, 1978, pp. 108 et ss.). Parce que Touraine s'est engagé à fonder une théorie générale de la sociologie, il s'est sourtout intéressé aux conflits de classes, dont les dimensions historiques étaient les plus évidentes: mouvement ouvrier dans la société industrielle, mouvement antitechnocratique dans la société postindustrielle. Cette politique l'aura conduit à négliger l'action des «nouveaux mouvements sociaux» qui nous intéressent ici (Touraine 1984, pp. 151157) ${ }^{1}$.

Actuellement, il est très difficile de prévoir l'importance «historique» de ces nouveaux mouvements. Mais leur impact politique est réel, en raison des modifications que leur existence ap-

1. En fait, Alain Touraine n'accepte de reconnaître comme «mouvement social» que les mobilisations collectives organisées, susceptibles de se constituer comme acteurs historiques principaux de la société postindustrielle. La question est de savoir quel mouvement social jouera, dans la société postindustrielle, le rôle que détenait le mouvement ouvrier dans la société industrielle. Dans ce cadre particulier, la notion de mouvement social est indissociable de la notion de classe sociale. Pour Touraine, les nouveaux mouvements dont il est question ici sont, au mieux, compris comme des mouvements culturels (le mouvement féministe) ou comme des acteurs politiques. Ils ne constituent pas, au sens tourainien du terme, un "acteur social historique», leur action se limitant à une série d'enjeux instrumentaux et de stratégies à l'égard du pouvoir politique. 
porte à l'équilibre des rapports institutionnels. D'ailleurs, les enjeux politiques ne sont pas tous de même nature. Pour les fins de cet exposé, nous en avons retenu trois, qui sont autant de conflits ou de façons particulières de définir les acteurs:

1) L'enjeu né de la répartition des ressources publiques qui, dans un premier temps, fait s'opposer les contribuables et les bénéficiaires des programmes sociaux-ici les payeurs de taxes et les personnes âgées- et, dans un deuxième temps, les bénéficiaires des différents groupes sociaux (par ex.: les jeunes et leurs aînés). Il pose dans des formes nouvelles le problème plus ancien de la répartition de la richesse collective et du conflit des intérêts particuliers.

2) L'enjeu associé à la reconnaissance politique du groupe social qui génère un conflit entre le «nouveau mouvement social» et les acteurs traditionnels du système politique (par ex.: les grandes associations de personnes âgées et les partis politiques). Il s'agit là essentiellement du problème de l'intégration de nouveaux agents représentatifs au sein d'un système institutionnel déjà constitué et en partie sclérosé.

3) L'enjeu entourant l'identification des porte-parole des personnes âgées, qui fait s'opposer entre elles des organisations désireuses de se constituer comme représentants de l'ensemble des âेnés. Il pose le problème de la reconnaissance des organisations représentatives et met en évidence les causes de leurs rivalités.

$\grave{A}$ chacune de ces questions correspond un «conflit» particulier qui met en «cause» des «acteurs rivaux» et les départage de part et d'autre d'un pôle qui touche directement les choix, le fonctionnement ou la composition des «centres de décision» politiques. D'ailleurs, ces conflits ne sont pas propres au mouvement des personnes âgées aux États-Unis; ils peuvent servir de cadre d'analyse des changements politiques que provoque immanquablement la mobilisation des nouvelles minorités. 


\section{L'action collective des personnes âgées aux États-Unis}

Les personnes âgées représentent aux États-Unis le groupe dont le nombre a augmenté le plus significativement au cours des 40 dernières années. De 12,4 millions qu'elles étaient en 1950, les personnes de 65 ans et plus sont passées à 25,7 millions en 1990, et représentent actuellement $12,4 \%$ de la population américaine. Dans l'hypothèse où les taux de mortalité et de fertilité se maintiendraient dans la moyenne, les personnes âgées pourraient constituer $21,1 \%$ de la population en l'an 2030 (Rhodes, 1988, pp. 4-7). Par ailleurs, cette population se compose en grande partie de femmes. Ainsi, en 1980, on comptait 68 hommes pour 100 femmes, chez les personnes de 65 ans et plus et 55 hommes pour $100 \mathrm{fem}$ mes parmi les individus de 75 ans et plus (Ibid., p. 10).

Plusieurs causes expliquent l'augmentation significative de cette tranche de la population américaine: taux de natalité élevé et fort taux d'immigration à la fin du XIXe siècle et au début du XXe siècle ; augmentation de l'espérance de vie; et déclin du «BabyBoom» (1945-1965): tous des éléments qui ont provoqué un renversement graduel de la pyramide des âges (Ibid., pp. 6 et 18).

Des mutations sociales importantes ont par ailleurs favorisé le regroupement des personnes âgées et leur ont fait prendre conscience d'une réalité commune: l'urbanisation accélérée de l'entre-deux guerres; l'instauration de la retraite, avec l'institution de la Sécurité sociale en 1935; l'apparition de la préretraite; l'appauvrissement relatif des personnes âgées et leur isolement social causés par leur retrait du marché du travail et par l'effritement de la famille traiditionnelle (Three generations families) (Pratt, 1976, p. 75).

Néanmoins, la mobilisation des personnes âgées est un phénomène encore récent. Ce n'est, en effet, qu'à la fin des années 50 que se constituent les premières associations permanentes de personnes âgées: l'American Association of Retired Persons (1958), le National Council of Senior Citizens (1961) et le Gray Panther (1970). Leur apparition dans l'espace public implique cependant un chambardement des priorités politiques et sociales; 
elle alimente de nouveaux conflits; et elle entraîne un renouvellement des agents politiques.

\section{Enjeu 1: la répartition des ressources entre les groupes sociaux}

L'avènement de nouveaux groupes sociaux, reconnus pour leurs besoins particuliers, pose le problème de la répartition des avantages sociaux accordés en vertu des programmes gouvernementaux de transferts. La question touche autant la nature des rapports entre les bénéficiaires et les contribuables que celle des rapports entre les différentes catégories de bénéficiaires.

\section{Le rapport bénéficiaires-contribuables}

Longtemps les personnes âgées ont été considérées comme un groupe intouchable. Qui oserait, en effet, affirmer que les divers régimes de pensions et autres prestations sont des avantages indus? Au cours des années 1935-1945, au lendemain de l'adoption du nouveau régime fédéral de la Sécurité sociale, les différents sondages réalisés auprès de la population américaine ont révélé un soutien indéfectible de l'opinion publique (Schiltz, 1970, pp. 3638) à cette cause. Cet enthousiasme devait en fait se maintenir jusqu'à la fin des années 70 .

Au début de la période Reagan, la part des budgets destinés au financement des programmes pour personnes âgées représente $27 \%$ des dépenses fédérales (Kosterlitz, 1987, p. 2652). Alors que la proportion des personnes âgées vivant au-dessous du seuil de pauvreté diminue, les aînés sont de moins en moins perçus comme les membres d'un groupe démuni. Petit à petit, on estime que les programmes sociaux dont ils bénéficient sont la cause principale du déficit budgétaire fédéral (Binstock 1983, p. 136). Dès le mileu des années 70 , on commence même à craindre un backlash de l'opinion publique à l'égard des personnes âgées (Reagan, 1977, p. 13). Cette manière de voir est entretenue par les médias natio- 
naux. Finalement, si un tel retournement n'a pas lieu, on enregistre une modification des priorités politiques des contribuables.

Si, tout au cours des années 80, la majorité des Américains reconnaît la nécessité de maintenir les programmes sociaux, un pourcentage élevé de personnes se montrent favorables à une augmentation éventuelle des dépenses fédérales en matière de pensions et de santé. Ainsi, les appuis accordés au principe de l'indexation des pensions passent, de $97 \%$ qu'ils étaient en 1974, à $72 \%$ en juin 1981 (Shapiro, Smith, 1985, p. 570). En mai 1982, 31\% des répondants se déclarent en faveur de l'abaissement du taux d'indexation (Ibid., p. 571). Entre 1982 et 1985, la proportion des personnes favorables au gel temporaire des pensions de vieillesse (retard dans l'indexation des pensions) passe, de 39\% en mars 1982, à 52\% en janvier 1983, avant d'atteindre 56\% en février 1985 (Ibid.). Entre juillet 1978 et mars 1981, de plus en plus de répondants jugent que le niveau des primes payées à la Sécurité sociale, soit $28 \%$ en 1978 et $36 \%$ en 1981 ( Ibid, p. 572), est trop élevé.

Toutes ces données convergent finalement: si une majorité de répondants se prononcent contre toute atteinte au régime des pensions, un nombre croissant de citoyens américains résistent à l'idée d'améliorer le régime. Plusieurs mettent en doute la nécessité de sa remise à jour périodique. L'intérêt des contribuables s'oppose donc en partie à celui des bénéficiaires.

Ce conflit apparaît nettement en 1983, à l'occasion de la réforme du régime de Sécurité sociale. Espérant à l'époque limiter le niveau croissant des primes destinées au financement des programmes de pension, le mouvement syndical (AFL-CIO) accepte le relèvement graduel (de 65 à 68 ans) de l'âge donnant droit à la pleine pension de vieillesse. En restreignant de la sorte l'accessibilité au régime, la réforme abaissait d'autant le niveau des primes payables par les travailleurs salariés (Clark, 1983) ${ }^{2}$. Pour les mê-

2. De ce point de vue, les personnes âgées ont eu plus de difficulté à préserver leurs acquis aux États-Unis qu'au Canada. Deux raisons semblent expliquer cet état de fait: l'effritement partiel du 
mes raisons, le mouvement syndical s'opposera au maintien des pensions de vieillesse en faveur des personnes âgées bénéficiant d'un revenu d'emploi (Pratt, 1976, p. 101). Et suivant une logique un peu semblable, de nombreuses associations de «payeurs de taxes» - de tendance plutôt conservatrice - soutiendront les politiques reaganiennes de réduction des programmes sociaux (Lesemann, 1988, pp. 109, et ss.).

\section{Le rapport entre les groupes sociaux de bénéficiaires}

Au-delà des inquiétudes éveillées par le coût des programmes sociaux qui risquaient d'engloutir une part considérable des budgets fédéraux, les années 80 révèlent l'opposition de plus en plus affirmée des différents groupes de bénéficiaires eux-mêmes.

Cette opposition est restée latente jusqu'au début de la décennie. Les associations de personnes âgées forment un lobby puissant qui sera perçu comme la cause principale des coupures imposées aux catégories sociales plus vulnérables (Vinyard, 1983, p. 1961). Des études de l'époque indiquent un appauvrissement relatif des jeunes ménages, entre 1968 et 1982, qui contraste avec la situation socio-économique relativement stable des personnes âgées (Duncan et al., 1986, pp. 26-33). Lentement, le Children's lobby va s'opposer au Gray lobby . La nécessité de contrecarrer le pouvoir politique des personnes âgées poussera même certaines associations à demander qu'on accorde aux parents un vote spécial pour chaque enfant à charge, de manière à renforcer le poids politique relatif des enfants (Binstock, 1983, p. 141; 1986, p. 129).

Poussant l'affrontement aux limites d'un conflit de générations, un nouveau groupe, The Americans for Generation Equity (ACE), ira jusqu'à prôner la limitation des programmes de Sécurité sociale et des programmes publics de santé afin de protéger les «Baby-Boomers» contre l'éventuel assèchement du fonds

support de l'opinion publique américaine relativement au régime de sécurité sociale, et l'unanimité des partis politiques au Congrès. 
fédéral des pensions (Trust fund) quand ils prendront à leur tour leur retraite. Ce nouveau discours allait frapper d'indignité les demandes traditionnelles des groupes nationaux de personnes âgées et forcer ceux-ci à se rapprocher des associations d'économie familiale et autres porte-parole de la jeune génération.

Quant aux personnes âgées, elles n'ont pas été elles non plus à l'abri des divisions internes. L'on a ainsi vu se multiplier les sous-groupes, aussi bien de femmes âgées (Older Women) que de membres âgés de la communauté noire (Black Aged) ou de la communauté hispanique, revendiquant tous un traitement particulier et la mise sur pied de programmes spécifiques (Lammers, 1983, pp. 59 et 212).

Les politiques reaganiennes allaient elles aussi provoquer d'autres conflits au sein du groupe social. Dans le cadre d'une réforme des responsabilités respectives du gouvernement fédéral et des États en matière de gestion et de financement des programmes sociaux (projet du New Federalism), le gouvernement fédéral sera appelé, au début des années 80 , à réduire la part des budgets nationaux jusque-là destinés au financement des programmes fédéraux. Leur gestion est confiée dorénavant à chaque État, auquel on accorde une enveloppe budgétaire unique (Lesemann, 1988, pp. 127 et ss.). Cette rationalisation des dépenses, qui pose clairement le problème du partage des budgets sociaux, a rapidement alimenté la confrontation des différents groupes de bénéficiaires, en faisant s'opposer les catégories sociales consacrées (les personnes âgées, par exemple) et les nouveaux groupes dans le besoin (truly needy): les pauvres, les malades, etc. (Achenbaum, 1983, p. 143). Dans certains cas, les conflits sont allés très loin. Ainsi, sur la Côte Ouest, à la suite des coupures imposées dans les programmes de transport adapté, on a vu s'opposer de façon particulièrement pitoyable, d'un côté les paraplégiques et, de l'autre, les handicapés visuels: deux groupes composés d'un grand nombre de personnes âgées (Peirce, Choharis, 1982, p. 1562).

Ce qu'il convient de retenir, c'est l'enjeu de la répartition des fonds publics entre les différents groupes sociaux et les conflits qui en résultent soit entre les bénéficiaires et les contribuables, soit entre les bénéficiaires eux-mêmes. Mais là n'est 
pas le seul problème que pose l'arrivée d'un nouveau groupe social dans l'espace public. La place réservée au groupe dans la définition des politiques publiques entraîne également de sérieuses difficultés. Tel est l'enjeu de la participation politique des nouvelles associations.

\section{Enjeu 2: la participation politique des nouvelles organisations}

L'affirmation de nouveaux groupes sociaux soulève le problème de leur participation au processus de définition des politiques publiques, particulièrement de celles qui les concernent directement. Or, cette participation met en cause la légitimité des instances politiques électives et la représentativité des agents politiques traditionnels: d'une part, la Présidence, le Congrès, les partis politiques et, d'autre part, les représentants d'intérêts socioéconomiques, devenus graduellement des «partenaires» dans le processus décisionnel, soit le mouvement syndical, les organisations agricoles, les entreprises, etc... La présence de nouveaux intervenants au sein du système menace en effet la place que plusieurs occupaient jusque-là comme agents reconnus de la représentation politique.

Pourtant, le système politique américain est considéré comme très perméable (Badie, Birnhaum, 1982, pp. 203 et ss.). Divers modes de participation ont permis une interpénétration extensive des domaines politique et civil:

- liberté d'action des lobbyistes;

- multiplication des Political Action Committees collaborant au financement des campagnes électorales nationales;

- participation des groupes aux audiences des comités et sous-comités du Congrès;

- participation aux nombreux comités de travail de la Maison Blanche et de l'administration publique (Task Force);

- présence de ces groupes dans des comités consultatifs permanents (Advisory Councils) établis dans toutes les sphères de l'action de l'État; 
- multiplication des contrats de services favorisant la participation des groupes dans la réglementation et l'instrumentation des politiques publiques, etc. (Schlozman, Tierney, 1986,189 et ss., et 322 et ss.).

Toutes ces pratiques ont apparemment permis aux groupes les plus importants de se faire valoir au sein du système politique, dans leur domaine particulier. On parlera alors de Subgovernment ou de Policy System, deux structures théoriques équivalentes, qui permettent de rendre compte de ces réseaux plus ou moins formels, où l'ensemble des acteurs d'un secteur donné est appelé à participer à la prise des décisions politiques sans trop se formaliser des frontières traditionnelles établies entre le législatif, l'exécutif et l'administratif (Vinyard, 1972, p. 281).

La réalité de ces Policy systems est cependant plus complexe qu'il n'y paraît de prime abord. Si la pratique du lobby et la participation aux audiences des comités législatifs fournissent aux groupes organisés un accès immédiat aux centres de décision, elles limitent souvent en contrepartie leur pratique aux formes d'influence les plus élémentaires. En fait, les structures formelles de la consultation sont occupées par d'autres agents politiques, souvent plus anciens. Les groupes socio-économiques en particulier, déjà reconnus au sein des différents Policy Systems, cherchent souvent à freiner l'arrivée de nouveaux groupes sociaux, ce qui s'est produit au sein de l'Aging Policy System. Aux États-Unis, en ce qui a trait à la population âgée, l'intervention historique des associations de professionnels, des organismes de services et de charité, des centrales syndicales et des représentants de l'industrie de la santé, a eu pour effet de limiter l'intervention des nouvelles associations de personnes âgées. Les intervenants apparus dans les cadres constitutionnels (Congrès, partis politiques et administration publique) ont parfois eux-mêmes résisté à l'engagement politique des nouvelles organisations. À cet égard, certains auteurs ont parlé du caractère «oligopolistique» du système politique américain (Lowi, 1987, pp. 86 et 106). Aux États-Unis comme dans la plupart des pays occidentaux, les acteurs politiques officiels réagissent assez différemment à l'action des nouveaux groupes sociaux qui parfois consolident leur position institutionnelle et parfois la menacent. 
Des stratégies d'ouverture

Jusqu'à l'élection de Ronald Reagan, la Maison Blanche s'est montrée plutôt favorable à l'intervention des nouveaux groupes. Les présidents américains ont vu généralement leur popularité s'effriter graduellement au lendemain des élections qui les avaient portés au pouvoir (Nadeau, Lachapelle, 1989). De ce fait, ces présidents ont souvent dû s'associer aux porte-parole des groupes les plus populaires dans l'opinion publique. Parallèlement, en raison du décalage des échéances électorales et de la souplesse des liens partisans, l'administration présidentielle entre souvent en conflit avec sa propre majorité au Sénat et à la Chambre des représentants (Poole, 1981, pp. 57-58). L'exécutif est par conséquent contraint de chercher auprès des groupes la légitimité qui lui permettra de s'imposer au Congrès. C'est donc souvent pour des raisons tactiques que la Maison Blanche a encouragé l'engagement politique des groupes organisés (Pratt, 1976, pp. 89-99; Lammers, 1983, pp. 71 et 231-132).

Ce fut notamment le cas au cours des mandats Kennedy et Johnson et si l'administration Nixon-Ford a d'abord eu tendance à résister à l'action des groupes nationaux de personnes âgées, elle en est venue elle aussi à compter sur leur complicité. C'est au cours des mandats Nixon qu'ont été mises sur pied les premières structures ad hoc de consultation des groupes et qu'ont été créés le Federal Council on Aging (FCoA), et, au niveau des états et des localités, une multitude de State and Local Councils on Aging. Mais c'est l'administration Carter qui a le plus systématiquement favorisé l'action des groupes nationaux en créant un poste de conseiller spécial à la Maison Blanche (Special Counselor on Aging), en favorisant la présence des porte-parole des groupes organisés au FCoA, en participant lui-même à certaines activités des groupes et en multipliant les alliances avec leurs représentants (Lammers, 1983, p. 72).

Le Parti démocrate, lui aussi, a servi de relais politique aux groupes organisés, mais non à la manière de l'administration présidentielle. Contrairement à la Maison Blanche, qui fait assez peu de cas des différentes tendances existant au sein du Senior Citizens 
Movement - et qui encourage la formation de coalitions diverses -, les démocrates ont cherché à s'associer à des regroupements déjà acquis à une vision «libérale» du problème des personnes âgées. L'action de ces associations sert ainsi en retour les objectifs électoraux du parti. En favorisant la reconnaissance de certaines associations plutôt que de certaines autres, les démocrates ont cependant attisé les rivalités qui existaient déjà entre les grandes organisations de personnes âgées.

Cette dynamique d'interaction est particulièrement bien illustrée dans la complicité qui unit traditionnellement le Parti démocrate et le National Council of Senior Citizens (NCSC), créé en 1961 à l'initiative de l'AFL-CIO. Le NCSC allait ainsi se faire le promoteur empressé du programme Medicare (1965), fortement soutenu par les démocrates. À partir de cette période, il a aussi pris part aux activités électorales du grand parti américain, soit en incitant régulièrement ses membres à voter pour les candidats démocrates aux élections législatives et aux élections présidentielles, soit en s'associant à l'organisation électorale du parti. En contrepartie, le NCSC a reçu le soutien des démocrates au Congrès, à la Maion Blanche, dans l'administration publique et pendant les décennies 60 et 70, auprès d'agences comme l'Office of Economic Opportunity, et auprès du Department of Health Education and Welfare (HEW) et du Department of Labor . D'autres associations, (l'American Association of Retired Persons (AARP) par exemple), parce qu'elles étaient moins proches des démocrates, ont souvent été arbitrairement identifiées au Parti républicain. Toutefois, le NCSC étant directement lié au Parti démocrate, une partie des personnes âgées d'allégeance républicaine sont devenues membres du AARP plutôt que du NCSC.

\section{Des stratégies de fermeture}

$\mathrm{Si}$, pendant longtemps, les administrations présidentielles successives et le Parti démocrate ont favorisé à leur façon l'action politique des groupes de personnes âgées (dont l'implication institutionelle servait leur propre intérêt politique et leur propre légi- 
timité), il en va tout autrement d'autres acteurs politiques qui se sont plutôt sentis menacés par l'activité politique des groupes nationaux.

C'est notamment le cas de l'administration publique fédérale. On pouvait prévoir que les grandes agences fédérales, qui avaient abordé jusque-là le dossier des personnes âgées sous l'angle des services publics, résisteraient à la participation de ces groupes au processus politique (Vinyard, 1983, p. 182). Aussi longtemps que les personnes âgées étaient restées confinées à leur statut de clientèle administrative, les grands responsables de la fonction publique avaient pu s'approprier entièrement, au sein de l'État, le rôle d'experts dans ce dossier. De ce point de vue, l'optique de l'administration s'opposait inévitablement à celle des groupes en quête de reconnaissance politique (Lammers, 1983, pp. 232-233).

En outre, les associations nationales ont de tout temps revendiqué l'institution d'une agence entièrement consacrée à la gestion des programmes sociaux destinés aux personnes âgées. Or, depuis la restructuration administrative imposée par le Report of the Commission on Organisation of the Executive Branch Government de 1949, l'administration publique américaine a plutôt encouragé le maintien d'un découpage «fonctionnel» des juridictions de chaque département, limitant du même coup la constitution d'agences «à clientèles» (Pratt, 1983, pp. 176-177). Le dossier des personnes âgées se trouve donc géré par une multitude de départements rivaux: Health, Education and Welfare (sécurité sociale et santé), Department of Labor (retraite et emploi), Department of Agriculture (alimentation), Department of Housing and Urban Developement (logements sociaux, résidences pour personnes âgées). Les programmes instaurés par ces agences étant surtout axés sur la production de services spécialisés, c'est souvent auprès des organisations de services (seniors centers, associations professionnelles et organisations privées de charité) qu'elles trouveront les alliés les plus intéressés (Estes, 1983, p. 63). Approche qui les a conduites à laisser de côté les groupes autonomes de personnes âgées, auxquels ces programmes étaient destinés. En 1965, sous l'impulsion de la Maison Blanche, la création de l'Administration on Aging au sein du HEW avait permis la con- 
centration d'un certain nombre de programmes spéciaux au sein d'une agence unique. L'incapacité du AoA de coordonner l'action des autres départements concernés et le maintien de l'agence à des paliers administratifs inférieurs allaient cependant limiter l'influence que les groupes nationaux auraient pu avoir sur l'orientation des programmes et des politiques publiques (Vinyard, 1978, p. 28; 1972, p. 293). Dans le même sens, les fonds réservés à des projets menés en dehors de l'agence fédérale, et qui auraient pu profiter aux groupes organisés, ont souvent été attribués en priorité aux agences administratives instituées par les États (State and Local Agencies on Aging ). Cette tendance devait limiter d'autant les rapports potentiels de l'AoA avec les groupes nationaux (Binstock, 1976, p. 226; Pratt, 1976, pp. 118 et 125).

Cette fermeture sera renforcée par l'administration Reagan (1981-1988) qui, désireuse de rétablir l'autonomie politique de l'État, a réduit au minimum les entrées institutionnelles dont auraient pu profiter les groupes organisés. En abolissant le poste de Special Counselor on Aging, en limitant la présence des porteparole des groupes au Federal Council on Aging et en diminuant les budgets du FCoA, la Maison Blanche manifestait sa volonté de rompre avec la pratique de concertation qui avait eu cours dans le passé (Lammers, 1983, pp. 72-73). Dans le même sens, l'administration présidentielle allait retirer aux groupes subventionnés le droit de participer aux audiences consultatives des différents comités du Congrès. Dans la foulée, l'administration publique a mis fin aux pratiques de consultation qu'elle avait timidement établies antérieurement. Les exemples de ce type se sont multipliés, laissant plus que jamais les groupes en marge des lieux de consultation et de décision.

De façon un peu différente, le Parti républicain, le Congrès et le mouvement syndical allaient résister eux aussi à l'action des organisations autonomes de personnes âgées. Contrairement à la fonction publique ou à la Maison Blanche (1981-1988), ce n'est pas au nom d'une expertise acquise - ou au nom de l'intégrité des instances politiques instituées - que ces agents politiques prétendent repousser l'envahisseur. C'est au nom de leur propre capacité à représenter le nouveau groupe social. En repoussant les 
initiatives des associations autonomes, l'administration publique et la Maison Blanche exprimaient surtout leur refus de reconnaître les personnes âgées en tant que groupe social spécifique. Elles niaient de ce fait la légitimité des organisations qui prétendaient parler en leur nom. Au contraire, le Parti républicain, le Congrès et l'AFL-CIO, tout en reconnaissant la spécificité du groupe social, se donnent pour leurs porte-parole. Dans tous les cas, ils ont tenté de créer au sein de leurs propres instances des structures d'accueil particulières destinées à encadrer l'action collective des personnes âgées. Leur initiative est ainsi venue concurrencer l'action des organisations nationales autonomes.

Les républicains, par exemple, ont toujours considéré les personnes âgées comme une clientèle captive, largement constituée d'électeurs conservateurs. Les sondages réalisés auprès des personnes âgées semblent du reste confirmer cette analyse (Light, 1981 , p. 2344). Dans cette foulée, le Parti républicain a rapidement mis sur pied sa propre structure d'accueil: The Republican National Committee of Senior Citizens. Il entretiendra peu de rapports avec les grandes organisations nationales, ce qui - dans ce dossier comme dans d'autres - distingue nettement l'approche du Parti républicain de celle du Parti démocrate.

Dans le même sens, les membres élus du Congès (Sénat et Chambre des représentants) ont été enclins à se présenter euxmêmes comme les porte-parole des personnes âgées qui constituent une partie importante de leur électorat. Chaque chambre a ainsi créé son propre comité: au Sénat, le Special Committee on Aging, et à la Chambre, le Select Committee on Aging (Vinyard, 1972, 1979). Ces deux comités sont l'expression de l'intérêt que portent les membres du Congrès à ce dossier. La préoccupation des sénateurs et représentants pour la cause des personnes âgées est d'autant plus intéressante que le Congrès a depuis quelques années essayé de limiter le nombre des comités et sous-comités «à clientèle» en favorisant les refontes «fonctionnelles» du système des comités. Le Congrès a ainsi évité de laisser aux groupes nationaux le soin d'occuper seuls tout le champ de la représentation, et certains «congressmen» ont aussitôt fait profession de défendre, au sein des instances législatives, l'intérêt des personnes âgées. 
Le mouvement syndical s'est lui aussi efforcé de conserver sa légitimité dans ce domaine. La question du vieillissement a souvent été réduite aux problèmes de la retraite et des pensions de vieillesse qui intéressent directement le mouvement syndical. Au cours des années 30, les personnes âgées sont d'abord et avant tout perçues comme d'anciens travailleurs ou comme les conjoints d'anciens travailleurs. Mais, à partir de leur retraite, les travailleurs cessent d'être membres de leur syndicat. Les syndicats industriels affiliés à l'AFL-CIO ont donc rapidement compris l'intérêt que représentait pour eux la création de structures d'accueil particulières pour leurs anciens travailleurs. La multiplication des Retired Workers Centers, au milieu des années 50, est le résultat de cette préoccupation (Pratt, 1976, p. 48). Parallèlement, le mouvement syndical conservait sa crédibilité en tant que porte-parole politique des personnes âgées. Au début des années 60, alors que se multiplient les associations nationales autonomes de personnes âgées, l'AFL-CIO contribua directement à mettre sur pied le National Council of Senior Citizens, qui fédère la majorité des Retired Workers Centers, avant d'associer à la mouvance syndicale d'autres structures constituées de façon autonome (Ibid., p. 89). On comprendra jusqu'à quel point cette pratique coupe l'herbe sous le pied des associations autonomes naissantes, en garantissant au mouvement syndical le maintien de sa légitimité représentative.

Au cours des dernières années, le NCSC a développé une forme d'autonomie relative par rapport aux organisations syndicales, en raison de sa capacité plus grande de se financer lui-même et de la diversité croissante de son «membership». Compagnon de voyage, le mouvement syndical va lentement changer sa stratégie, qui ressemble de plus en plus aujourd'hui à celle du Parti démocrate: plus souple et moins hégémonique.

\section{Enjeu 3: le choix politique des porte-parole des personnes âgées}

L'avènement de nouveaux groupes sociaux engendre un dernier problème politique: le choix des groupes «les plus repré- 
sentatifs» parmi tous ceux qui prétendent agir et parler au nom des citoyens âgés. Dans le cadre des démocraties libérales pluralistes, la «représentativité» est devenue la mesure de la légitimité politique des instances électives, aussi bien que des agents de la société civile qui, au cours des années, ont réussi à s'intégrer au processus politique décisionnel. Il en va de même des nouveaux groupes sociaux.

Au milieu des années 80 , on compte près de mille organisations, dont plusieurs peuvent prétendre, à un titre ou à un autre, agir au nom des personnes âgées (Pratt, 1983, p. 146). De ce fait, chaque association cherche à revendiquer la reconnaissance du caractère représentatif de son discours, de ses pratiques et de son membership. La «représentativité» devient par conséquent l'enjeu d'un conflit larvé entre les associations les plus importantes, qui prétendent toutes l'emporter sur les autres au jeu des légitimités. Il existe tout de même quelques exceptions à cette règle. Les groupes de moyenne dimension comme le Gray Panther -60000 membresont toujours favorisé la création de grandes coalitions nationales. Les organisations plus importantes, comme le National Council of Senior Citizens (4,5 millions de membres) et l'American Association of Retired Persons (30 millions de membres), se sont fait concurrence en matière de représentativité politique. Et la lutte que ces associations se livrent est d'autant plus virulente que les espaces formels de participation sont rares. Cette situation favorisant d'abord la guerre des memberships, chacune des associations s'est prétendue la plus importante ou la plus connue, et partant, la plus capable de représenter les personnes âgées: The largest association representing Older Americans (AARP), ou The largest and best known organisation of mature persons' clubs (NCSC).

Il en est ainsi de l'effort que ces associations ont fait pour distiguer leurs revendications de celles de leurs concurrentes. Parce que chaque organisation puise à des bassins de mobilisation différents, le profil de leur membership s'est lentement particularisé. Pour des raisons historiques évoquées ci-dessus, le membership du NCSC est composé surtout d'anciens travailleurs manuels -syndiqués- du secteur industriel et du Nord-Est américain. Il se différencie en cela du membership du AARP, constitué princi- 
palement de retraités des classes moyennes et moyennes supérieures, anciens cols blancs et anciens professionnels. Ces profils se sont perpétués et ont déterminé pour chaque association le choix de priorités politiques différentes, et la définition de positions concurrentes sur une multitude de questions inscrites au programme politique (Pratt, 1976, p. 101). De cette façon, chaque organisation présente son interprétation de la réalité comme la plus apte à rendre compte des difficultés vécues - et des espoirs entretenus- par les aînés.

C'est pourquoi, au cours de la période 1961-1965, le NCSC appuie largement le nouveau projet de programme Medicare, et cela contre les positions du AARP qui préfère un programme privé d'assurance-santé. Plus tard, en 1971, le AARP se prononce en faveur du versement entier des pensions de Sécurité sociale aux personnes âgées travaillant après l'âge de 65 ans, alors que le NCSC se déclare plutôt en faveur d'une réduction proportionelle des pensions en fonction des revenus d'emploi de ces travailleurs. En 1978, le AARP favorise l'abolition de la retraite obligatoire que le NCSC propose de maintenir. En 1983, le AARP dénonce la réforme du régime de Sécurité sociale, approuvée conjointement par le Congrès et la Maison Blanche, et s'oppose en cela au NCSC qui appuie publiquement. À la faveur de ces divergences, des acteurs politiques traditionnels pourront se libérer partiellement de l'influence des groupes organisés, incapables de faire entre eux l'unanimié. Chaque organisation, en cherchant à affirmer sa spécificité, accentuera à sa manière la division du mouvement.

Même division sur le plan de la pratique politique. Le NCSC , proche du mouvement syndical, s'est souvent opposé aux modes d'action du AARP, plus ou moins associé au monde des affaires en raison des services importants qu'il offre à ses membres. Professant la combativité des personnes âgées, le NCSC aura plus souvent recours aux grandes manifestations de masse, et n'hésitera pas à forcer la main à certains Congressmen en leur rappelant l'importance de son membership dans leur district. Le AARP, plus conciliant, et dont le membership est moins militant mais plus imposant, jouera plutôt la carte de la force tranquille, quitte à accepter des compromis tactiques que n'assument pas les 
autres associations: Our responsibility grows with our size (Kosterlitz, 1978, p. 2653). Cet état de fait conduit parfois ces organisations à entretenir des alliances différentes qui les font s'opposer encore davantage. C'est ainsi que le NCSC a pu profiter des entrées de l'AFL-CIO auprès du Department of Labor . Il s'est rapidement vu engagé, par cette voie, dans la cogestion d'un ensemble de programmes d'emploi et de soutien aux travailleurs à la retraite, mis sur pied par le DOL. Le AARP, au contraire, a cherché à favoriser l'autonomie de l'Administration on Aging, et, en contrepartie, a favorisé le rapatriement de certains programmes au sein de l'AoA (Pratt, 1976, p. 146). Ce faisant, plusieurs agences ont trouvé un relais solide dans l'une ou l'autre de ces grandes associations rivales.

Au cours des dernières années, on a vu naître d'autres associations nationales importantes comme la National Alliance of Senior Citizens, plutôt conservatrice, et le National Committee to Preserve Social Security and Medicare, rapidement connu pour son discours alarmiste. Dans tous les cas, le problème reste cependant le même. En cherchant à se distinguer des autres associations, ces organisations entretiennent une compétition qui fait perdre au mouvement son unité d'action. La «guerre des memberships», les pratiques et les alliances concurrentes, les revendications divergentes, tous ces éléments viennent lentement à bout de ces entreprises collectives. Si l'histoire récente laisse voir quelques occasions de rapprochement entre les associations, notamment au début de la période Reagan, alors que l'intégrité du régime de Sécurité sociale est menacée, les prétentions hégémoniques de chacune ont plutôt effet de saper les bases d'une véritable concertation.

\section{Conclusion}

La mobilisation de nouveaux groupes sociaux engendre une modification des rapports politiques. Elle favorise le conflit entre les payeurs de taxes et les bénéficiaires de programmes sociaux; elle alimente les intérêts qui opposent les différentes catégories de 
bénéficiaires; elle fait naître de nouveaux conflits entre les nouvelles organisations représentatives et les anciens agents de la représentation politique; et elle provoque une compétition virulente entre ces organisations, elles-mêmes toutes en quête de légitimité politique. Tous ces rapports d'opposition sont fondés sur autant d'enjeux particuliers: partage de ressources publiques limitées; répartition des espaces politiques entre les acteurs anciens et nouveaux; choix des porte-parole de ces nouvelles minorités. L'action collective des personnes âgées aux États-Unis constitue un exemple particulier, mais non exclusif, des dynamiques qui viennent changer les rapports politiques, soit en transformant d'anciennes clientèles administratives en nouveaux intervenants politiques, soit en favorisant la participation institutionnelle de nouveaux agents politiques, ou encore en déclenchant entre ces nouveaux agents des conflits inconnus jusque-là.

L'avènement probable d'une mobilisation des aînés au Québec pourrait entraîner des effets semblables (Pérodeau, 1989). L'apparition d'autres groupes sociaux a déjà provoqué des réactions similaires. Une étude de l'action politique des groupes de jeunes au Québec que nous terminons actuellement s'inspire du même schéma interprétatif.

Cette perception particulière du renouvellement du champ politique ouvre donc la voie à une réflexion plus large sur les nouvelles formes de participation politique et sur la multiplication des espaces de négociation intitutionnels, dimensions que nous n'avons pas abordées mais qui pourraient devenir le point de départ d'une recherche ultérieure sur l'étanchéité de nos systèmes institutionnels et sur la diversité des sources de la légitimité politique dans les démocraties contemporaines. 


\section{Bibliographie}

ACHENBAUM, W.A., Shade of Gray: Old Age American Values and Federal Policies since 1920, Boston and Cie, 1983.

BADDIE, B., BIRNBAUM, P., Sociologie de l'État, (2e édition), Paris, Grasset, 1982, 204 pages (1re édi. française, 1979).

BINSTOCK, R.H., «Interest-group Liberalism and the Politics of Aging», dans Atchley, R.C., Seltzer, M.M., The Sociology of Aging: Selected Readings, Belmont (Cal.) Wadsworth Publishing, 1976, pp. 206-232.

BINSTOCK, R.H., «The Aged as Scapegoat», dans The Gerontologist, 1983, vol. 23, no. 2, avril, pp. 136-143.

BINSTOCK, R.H., «Public Policy and the Elderly», dans Geriatric Psychiatry, 1986, vol. 19, no. 2, pp. 115-143.

BOURASSA, G., «Les groupes d'intérêts», dans Orban, E., Le système politique des États-Unis, Montréal, Les Presses de l'Université de Montréal, 1987, pp. 91-111.

CLARK, T.B., «Congress Avoided Political Abyss by Approving Social Security Changes», dans National Journal, vol. 15, no. 12, 19 mars 1983, pp. 611-615.

DION, L., Le bill 60 et la société québécoise, Montréal, Édition HMH, 1967, 197 pages.

DION, L., Société et politique: La vie des groupes, (Tome 1: Fondements de la société libérale), Québec, Presses de l'Université Laval, 1971.

DUNCAN G.J., HILL, M., RODGER, W., «The Changing Fortune of Young and Old», dans American Demographics, 1986, vol. 8, n 8, pp. 26-33.

ESTES, C.L., et al., The Aging Enterprise (4e édition), San Francisco, Jossey-Bass Publishers, 1983.

KLEMMACK, D.L., «Public Support for Government Energy Assistance Payments to Older Persons», dans The Gerontologist, 1983, vol. 23, $\mathrm{n}^{\circ} 3$, pp. 307-312.

KOSTERLITZ, J., «Test of Strength», dans National Journal, 1987, vol. 19, n 43, 24 octobre, pp. 2652-2657. 
LACROIX, B., «Conclusion», dans D'Arcy F. (Ed.), La Représentation, Paris, Economica, 1985, pp. 175-185.

LAMMERS, W.W., Public Policy and the Aging, Washington D.C., C.Q. Presses, 1983.

LESEMANN, F., La politique sociale américaine, Montréal, Éditions Saint-Martin, Syros Alternatives, 1988.

LIGHT L., "Growing Prize at the Polls: Democrats Hoping to Break Traditional GOP Loyalties of Voters Age 65 and Older», dans Congressional Quarterly Report, 1981, vol. $39, \mathrm{n}^{\circ} 48,28$ novembre, pp. 2343-2346.

LOWI, T.J., La deuxième république des États-Unis: La fin du libéralisme, Paris, Presses Universitaires de France (coll. Recherches politiques), 1987, (1re édition américaine, 1969, 2e 1978).

NADEAU, R., LACHAPELLE, G., «Facteurs explicatifs des fluctuations de la popularité du président Reagan», dans Politique, 1989, $\mathrm{n}^{\circ}$ 16, pp. 35-58.

PEIRCE, N.R., CHOHARIS, P.C., «The Elderly as a Political Force: 26 millions Strong and Well Organized», dans $\mathrm{Na}$ tional Journal, 1982, vol. 14, $\mathrm{n}^{\circ} 37,11$ septembre, pp. 1559-1562.

PÉRODEAU, G., «Vieillir au Québec: d'une génération à l'autre», dans Santé Mentale au Québec, 1989, vol. 14, $\mathrm{n}^{\circ} 1$, pp. 191-198.

PIZZORNO, A., «Interest and Parties in Pluralism», dans Berger, S. (Ed.) Organizing Interest dans Western Europe: Pluralism, Corporatism, and the Transformation of Politics, Cambridge, London, Cambridge University Press, 1989, pp. 247-283.

POOLE, K.T., «Dimensions of Interest Group Evaluation of the U.S. Senate, 1969-1978», dans American Journal of Political Science, 1981, vol. 25, $\mathrm{n}^{\circ}$ 1, février, pp. 49-67.

PRATT, H.J., The Gray Lobby, Chicago, The University of Chicago Press, 1976.

PRATT, H.J., «National Interest Groups among the Elderly: Consolidation and Constraint», dans Browne, W.P., Olson, L.K., Aging and Public Policy: The Politics of Growing 
Old in America. Westport (Conn.), Greenwood Press, 1983, pp. 144-179.

RAGAN, P.K., «Another Look at the Politicizing of Old Age:

Can we Expect a Backlash?», dans The Urban \& Social Change Review, 1977, vol. 10, $\mathrm{n}^{\circ} 2$, pp. 6-12.

RHODES, C., An Introduction to Gerontology, Aging in American Society, Springfield (Illin.), Charles C. Thomas Pub., 1988.

SCHILTZ, M.E., Public Attitudes Toward Social Security: 19351965, Washington D.C., U.S. Department of Health, Education and Welfare, 1970.

SCHLOZMAN, K., Lehman, Tierney J.T., Organized Interests and American Democracy, New York, Harper \& Row Publishers, 1986.

SHAPIRO, R.Y., SMITH, T., «The Polls: The Social Security», dans The Public Opinion Quarlerly, 1985, vol. 49, $\mathrm{n}^{\circ} 4$, pp. 561-572.

TARROW, S., «National Politics and Collective Action: Recent Theory and Research in Western Europe and the United States», dans Scott, W.R., et Blake, J. (Eds.), Annual Review of Sociology, 1988, vol. 15, pp. 421-440.

TOURAINE, A., La voix et le regard, Paris, Seuil (Coll. Sociologie permanente), 1978.

TOURAINE, A., Le retour de l'acteur, Paris, Fayard (Coll. Mouvement), 1984.

VINYARD, D., "The Senate Committee on the Aging and the Development of a Policy System», dans Michigan Academician, 1972, vol. 5, $\mathrm{n}^{\circ} 2$, pp. 281-294.

VINYARD, D., «The Rediscovery of the Elderly», dans Society, 1987, vol. 15, n 5, pp. 24-29.

VINYARD, D., «The House Select Committee on the Aging», dans Long Term Care \& Health Services Administration", 1979, vol. 3, n 4, pp. 317-324.

VINYARD, D., «Public Policy and Institutional Politics», dans Browne W.P., Olson L.K., Aging and Public Policy: The Politics of Growing Old in America, Westport (Conn.), Greenwood Press, 1983, pp. 181-199. 
NOUVEAUX GROUPES SOCIAUX...

WEIGERT, A.S., TEITGE, D.W., TEITGE, J.S., Society and Identity, Cambridge, Cambridge University, 1986. 